\title{
Mindfulness in the Emergency Department (MED): An Asynchronous Learning Course to Practise Mindfulness and Resilience in the Emergency Room of Low Resource Setting
}

\author{
Syed Maaz Salahuddin and Shahan Waheed \\ Department of Emergency Medicine, The Aga Khan University Hospital, Karachi, Pakistan
}

\begin{abstract}
Owing to the fast-paced, stressful, and frequent crucial rapid decisions that trigger cognitive exhaustion, emergency physicians are especially vulnerable to burnout. Mindfulness practices have been defined in the literature and it focuses on the well-being and resilience. Mindfulness is characterised as the ability to use tools and expertise to mentally respond to environmental problems to fulfil psychological needs. To overcome burnout, a self-directed learning course was developed for emergency physicians that will include learning goals on mindfulness, peer group discussions and tasks, role playing and practising meditation exercises. It would concentrate on the foundations of mindfulness and resilience, learning and incorporating mindfulness, understanding the coping mechanisms and establishing a pathway to minimise anxiety and depression.
\end{abstract}

Key Words: Mindfulness, Resilience, Burnout, Emergency medicine, Emergency department, Wellness.

How to cite this article: Salahuddin SM, Waheed S. Mindfulness in the Emergency Department (MED): An Asynchronous Learning Course to Practise Mindfulness and Resilience in the Emergency Room of Low Resource Setting. J Coll Physicians Surg Pak 2021; 31(10):1242-1243.

Physician burnout and mental illness is a recognised, widespread, global phenomenon occurring in emergency medicine (EM) practice. It is marked by cognitive fatigue, depersonalisation, and a reduction in professional effectiveness. The reasons are multifactorial but, in a low middle-income country, these problems are much increased. ${ }^{1}$ In 2015, Shanafelt et al. conducted a study that showed that emergency physicians and primary healthcare providers had the highest rates of burnout. ${ }^{2}$ Hoonpongsimanont et al. concluded that emergency medicine residents, during their first year of residency, expressed disappointment with their contemporary lifestyle. ${ }^{3}$ The study also suggested that residency programme directors should incorporate healthy coping mechanisms to deal with EM residents' burnout. ${ }^{3}$ According to a recent survey, over $72 \%$ of emergency physicians had burnout. ${ }^{4}$ Mid-residency was the most common time of burnout, compassion fatigue, and workplace dissatisfaction. ${ }^{4}$ These issues are surmounted additionally due to lack of properinfrastructure and scarcity of qualified healthcare professionals.

Correspondence to: Dr. Syed Maaz Salahuddin, Department of Emergency Medicine, The Aga Khan University Hospital, Karachi, Pakistan

E-mail: maaz.salahuddin@hotmail.com

Received: April 14, 2021; Revised: July 05, 2021;

Accepted: July 14, 2021

DOI: https://doi.org/10.29271/jcpsp.2021.10.1242
The working environment of the emergency department harbingers a lot of factors that culminate in mental illnesses; and healthcare workers in the emergency department are regularly subject to high levels of workplace stress. The stressors that are worth mentioning are over-crowding due to limited healthcare workforce, increasing number of critical patients presenting to the emergency room, coping with mortality, resuscitation, dealing with mass casualties in a resource-limited setting, sleep deprivation, lengthy working hours and irregular duty schedule that exhausts a healthcare worker physically, mentally, and emotionally. Such stressors can produce detrimental health effects, resulting in emotional instability (pessimism, hopelessness and doubts), and loss of interest in routine activities, vicarious traumatisation, a feeling of lack of accomplishment, and even suicides. ${ }^{3,4}$

Therefore, to maintain a healthy and normal brain function, the physicians need to instill some refreshing activities into their work routine. ${ }^{5}$ Moreover, to overcomethis, a multidisciplinary collaboration at all levels of the healthcare system is required. A two-step approach that incorporates personally oriented solutions and systemic drivenstrategies was recommended to preventED physicians' burnout. ${ }^{5}$ Mindfulness helps in maintaining present-moment awareness; whereas, resilience aids in adapting to change and recovering from adversity. Mindful persons have a higher level of psychological resilience, and according to a growing body of evidence, mindfulness promotes resilience. ${ }^{6}$ Resilient people have a solution-oriented mindset, which, when combined with mindfulness, provides a sense of fulfilment and improves subjective well-being. ${ }^{6}$ 
Mindfulness in ED is an important issuethat has not been addressed in most of the emergency medicine training programmes of developing countries, like Pakistan. Hence, it was imperative to use evidence-based solutions that are readily available, realistic, and sustainable. In developing countries, there is a scarcity of emergency medicine residency programme and institutional tools for healthcare workers mindfulness and resilience. Considering the problem that could affect both physicians and their work, a course called Mindfulness in the Emergency Department(MED), wasdeveloped. The course will be introduced at the beginning of the emergency medicine residency programme. Thiscourse will utilise mindfulness-based interventions (MBI), mindfulness-based stress reduction (MBSR), and resilience building strategies to encourage wellness, perseverance and resilience among emergency medicine trainees. The MBSR combines meditation on attentiveness, bodily awareness, yoga, thinking patterns and individual's behaviour.

The MED course includes fundamentals on mindfulness in ED (activity I):, exercising mindfulness in ED (activity II: MBI), incorporating mindfulness and resilience strategies (activity III: $\mathrm{MBI}$ ), and session by a psychotherapist/psychologist (activity IV: MBSR) (Figure 1).

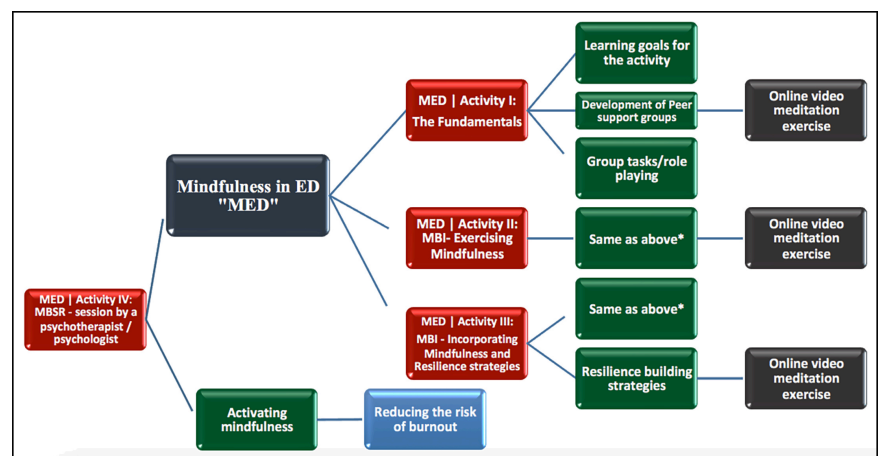

Figure 1: Flow chart/MED - content outline with instructional objectives. *(Learning goals for the activity, peersupport group discussions and grouptasks/role playing).

This self-directed learning course is structured to keep attendees engaged during lectures, meditation exercises and group discussions to help pursue mindfulness. Resilience enhancing activities will be introduced, so that emergency medicine physicians are able to cope up with changing emergency department environment and tough situations at work. Each attendee in this course will be expected to perform meditation exercises, according to the instructions of an online video. Weekly tasks will be assigned to all participants to ensure mindfulness at home and work, with emphasis on recognising stressors, continuing mindfulness activities, and outcomes of any progress observed. Journalling, documenting narratives, psychological self-assessment and peer mentorship, as well as spending time for calming activities, are all strategies that will be utilised in MED course to aid development of resilience in EM physicians. At the end of this course, the attendee will be given a feedback form; and a survey will be conducted to assess positive change in EM trainees' stress, burnout and resilience.
Piloting and documenting the benefits of MED course is necessary to make this curriculum sustainable. Various meditation applications are available; and one must give time to mindfulness exercises. Resilience amongst EM trainees has emerged as an integral part of their curriculum. Adequately funded projects are essential to assess the utility of mindfulness and resilience programmes in emergency medicine education. Thus, in conclusion, well-being policy must be established, and brief mindfulness sessions should be instilled in healthcare workers' wellbeing institutional policy. This will ensure the maximum working capacity of an individual, reducing the rising burnout, understanding their coping mechanisms, and establishing a pathway to minimise anxiety and depression.

\section{CONFLICT OF INTEREST:}

The authors declared no conflict of interest.

\section{AUTHORS' CONTRIBUTION:}

SMS: Conceptualisation of the study, "short communication" write-up, and critically reviewed the "short communication" as submitted.

SW: Conceptualisation and drafting the course outline, critically reviewed the "short communication" assubmitted.

\section{REFERENCES}

1. Global burden of disease 2017, disease and injury incidence and prevalence collaborators. global, regional, and national incidence, prevalence, and years lived with disability for 354 diseases and injuries for 195 countries and territories, 1990-2017: A systematic analysis for the global burden of disease study 2017. Lancet 2018; 392(10159):1789-858. doi: 10.1016/S0140-6736(18)32 279-7.

2. Shanafelt TD, Hasan O, Dyrbye LN, Sinsky C, Satele D, Sloan J, et al. Changes in burnout and satisfaction with work-life balance in physicians and the general US working population between. 2011 and 2014. Mayo Clin Proc 2015; 90(12):1600-13. doi: 10.1016/j.mayocp.2015.08.023.

3. Hoonpongsimanont W, Murphy M, Kim CH, Nasir D, Compton S. Emergency medicine resident well-being: stress and satisfaction. Occup Med (Lond) 2014; 64(1): 45-8. doi: 10.1093/occmed/kqt139.

4. Chung A, Felber R, Han E, Mathew T, Rebillot K, Likourezos A. A targeted mindfulness curriculum for medical students during their emergency medicine clerkship experience. West J Emerg Med 2018; 19(4): 762-6. doi: 10.5811/westjem.2018.4.37018.

5. West CP, Dyrbye LN, Erwin PJ, Shanafelt TD. Interventions to prevent and reduce physician burnout: a systematic review and meta-analysis. Lancet 2016; 388(10057): 2272-81. doi: 10.1016/S0140-6736(16)31279-X

6. Bajaj B, Pande N. Mediating role of resilience in the impact of mindfulness on life satisfaction and affect as indices of subjective well-being. Pers Individ Dif 2016; 93:63-7. doi.org/10.1016/j. paid.2015.09.005. 